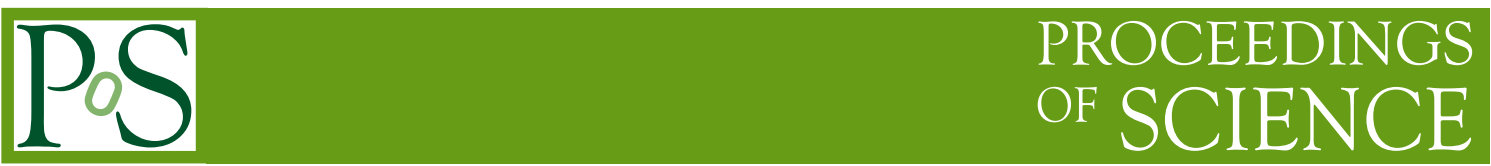

\title{
Overlap fermion with the topology conserving gauge action
}

\author{
Hidenori Fukaya $^{a}$, Shoji Hashimoto $^{b, c}$, Takuya Hirohashi $^{d}$, Hideo Matsufuru$^{* b}$ \\ Kenji Ogawa $^{c}$, and Tetsuya Onogi ${ }^{a}$ \\ ${ }^{a}$ Yukawa Institute for Theoretical Physics, Kyoto University, Kyoto 606-8502, Japan. \\ ${ }^{b}$ High Energy Accelerator Research Organization (KEK), Tsukuba 305-0801, Japan. \\ ${ }^{c}$ School of High Energy Accelerator Science, The Graduate University for Advanced Studies \\ (Sokendai), Tsukuba 305-0801, Japan. \\ ${ }^{d}$ Department of Physics, Kyoto University, Kyoto 606-8502, Japan. \\ E-mail: hideo.matsufurulkek.jp
}

\begin{abstract}
We investigate the distribution of low-lying eigenmodes of hermitian Wilson-Dirac operator, $H_{W}$, with the gauge action whose form is designed to avoid topology change, as well as with the standard plaquette action. On the quenched lattices, the former gauge action exhibits less density of low-lying eigenmodes of $H_{W}$ compared to the latter at the same lattice spacing. We also show preliminary results for the dynamical simulation with two flavors of overlap fermions.
\end{abstract}

XXIIIrd International Symposium on Lattice Field Theory

25-30 July 2005

Trinity College, Dublin, Ireland

* Speaker. 


\section{Introduction}

Recent progress of the chiral fermions, both in theoretical understanding and in technical development of numerical algorithms, is making its dynamical fermion simulation more feasible with the next generation machines. Wide range of applications from flavor physics to hadron physics is expected with the dynamical fermions having exact chiral symmetry.

The Neuberger's overlap-Dirac operator with mass $m$ is written as [1]

$$
D_{o v}(m)=\left(1-\frac{\bar{a}}{2}\right) D_{o v}+m, \quad D_{o v}=\frac{1}{\bar{a}}\left[1+\gamma_{5} \operatorname{sign}\left(H_{W}\right)\right],
$$

where $\bar{a} \equiv a /(1+s)$. For the kernel $H_{W}$ we use the Wilson-Dirac operator $D_{W}$ as

$$
H_{W}=\gamma_{5}\left[D_{W}-(1+s)\right] .
$$

The parameter $s$ is chosen in the range $|s|<1$. The locality of the overlap operator is proven when the bulk of eigenvalues of $H_{W}$ is bounded in a non-zero region [2]. This condition is satisfied when the gauge configuration is sufficiently smooth. In practice, however, it is known that there is non-zero density of near-zero eigenvalues when the gauge configurations are generated with the conventional Wilson gauge action [3]. Although the problem of locality is avoided if the near-zero modes are localized [4, 5, 6], it is more desirable if the gauge action does not generate such nearzero modes from the beginning. The use of improved actions (Lüscher-Weisz, Iwasaki, DBW2) is a popular choice, with which the density of the near-zero modes is reduced, but in this work we consider an alternative solution.

Since the zero mode of $H_{W}$ appears when the topological charge of the gauge field changes its value, we expect that the appearance of the near-zero mode is suppressed if we use a gauge action for which the topology change is suppressed or even forbidden. In this work, we employ the Lüscher's topology conserving gauge action [7] and investigate how much it suppresses the near-zero modes.

The suppression of topology change and of the appearance of the near-zero modes is desirable also in a practical point of view. (1) The cost of approximating the sign function in (1.1) is reduced; (2) In the dynamical fermion simulation, one can avoid the "reflection-refraction" process [8], which is very costly; (3) Gauge configurations with a fixed topological charge is efficiently accumulated, which is useful for the study of the $\varepsilon$-regime of chiral perturbation theory.

\section{Topology conserving gauge action}

The topology conserving gauge action can be constructed by enforcing the admissibility condition

$$
\left|1-P_{\mu v}(x)\right|<\varepsilon, \quad \forall x, \mu, v,
$$

where $P_{\mu v}(x)$ is the plaquette. $\varepsilon$ is a parameter to control the smoothness of the gauge field. For the SU(3) gauge theory, the locality of the overlap-Dirac operator is guaranteed when $\varepsilon \lesssim 1 / 20.49$ [9]. In practice this condition is too strong to realize the lattice spacing $\sim 0.1 \mathrm{fm}$ and we have to relax it to $\varepsilon \sim O(1)$. Therefore, the question is whether the good property is kept enough for these large value of $\varepsilon$. 


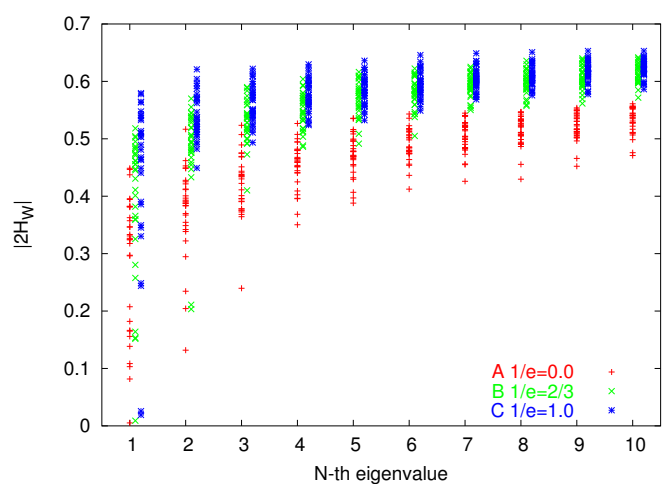

Figure 1: Low-lying eigenvalue distribution on quenched $16^{4}$ lattices for $1 / \varepsilon=0,2 / 3$, and $1\left(a^{-1} \simeq 2.5\right.$ $\mathrm{GeV})$.

An example of the gauge action to enforce (2.1) is

$$
S_{G}= \begin{cases}\frac{1}{g^{2}} \sum_{x, \mu, v} \frac{S_{P}(x)}{\left(1-S_{P}(x) / \varepsilon\right)^{\alpha}} & \text { if admissible } \\ \infty & \text { otherwise }\end{cases}
$$

where $S_{P}(x)=1-\operatorname{Re} \operatorname{Tr} P_{\mu v}(x) / N_{c}$. In this work, we set $\alpha=1$. Previously, this gauge action was used by two of us for the study of the massive Schwinger model [10]. In the four-dimensional QCD, Bietenholz et al. are also studying its property [11, 12].

In the following, we investigate the eigenvalue distribution of $H_{W}$ on the gauge configurations generated with this action. The topology change and scaling property of this action is presented in these proceedings [13].

\section{Quenched results}

First we examine the low-lying eigenvalue distribution of $H_{W}$ for the topology conserving gauge action in the quenched approximation. The configuration is generated with the Hybrid Monte Carlo algorithm with $\delta t=0.01$ and a length of trajectory 0.2 . The parameter $\varepsilon$ is set to $1 / \varepsilon=0$, $2 / 3$, and 1 . ( $1 / \varepsilon=0$ corresponds to the standard plaquette action.) The admissibility condition, $1-S_{P}(x) / \varepsilon>0$, is monitored during the molecular dynamics evolutions, and no violation has been found for $1 / \varepsilon=2 / 3$ and 1 . For finite $1 / \varepsilon$, while the topology change is not exactly prohibited, it is indeed much suppressed compared to the plaquette action $1 / \varepsilon=0$ [13].

Figure 1 shows ten lowest-lying eigenvalues of $\left|H_{W}\right|$ on a $16^{4}$ lattice for the three values of $\varepsilon$. The inverse lattice spacing determined with the Sommer scale is $a^{-1} \simeq 2.5 \mathrm{GeV}$ for each lattice. In the plot, $\mathrm{A}, \mathrm{B}$, and $\mathrm{C}$ correspond to the combinations $(\beta, 1 / \varepsilon)=(6.13,0),(2.70,2 / 3)$, and $(1.42,1)$, respectively. As expected, we find that the eigenvalue distribution shifts upward as $1 / \varepsilon$ increases.

Figure 2 shows the results on $20^{4}$ lattices. In this figure, the low-lying eigenvalue distribution is compared at five sets of parameters: $(\beta, 1 / \varepsilon)=(6.0,0),(2.55,2 / 3),(1.3,1),(2.7,2 / 3)$, and $(1.42,1)$. The first three correspond to $a^{-1} \simeq 2.1 \mathrm{GeV}$, while the last two are $2.6 \mathrm{GeV}$ and $2.5 \mathrm{GeV}$. The same tendency as in Figure 1 is found. 


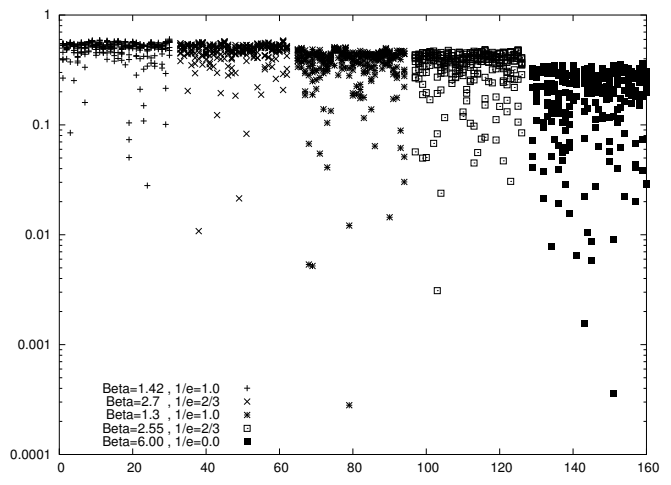

Figure 2: Low-lying eigenvalue distribution of $2 \mathrm{H}_{W}$ on quenched $20^{4}$ lattices.

\begin{tabular}{ccccc}
\hline \hline size & $\beta$ & $1 / \varepsilon$ & reflection/trj & acceptance \\
\hline $4^{4}$ & 5.40 & 0 & 0.34 & 0.97 \\
& 5.50 & 0 & 0.53 & 0.94 \\
\hline $4^{4}$ & 0.70 & 1 & 0.63 & 0.82 \\
& 0.80 & 1 & 0.18 & 0.89 \\
\hline \hline
\end{tabular}

Table 1: Parameters of our dynamical simulation runs.

We conclude that in the quenched calculation the density of the near-zero eigenvalues is smaller for the topology conserving gauge action than that of the plaquette action. This trend is enhanced as $1 / \varepsilon$ increases.

\section{Dynamical results}

In this section, we show the results of an exploratory study of the low-lying eigenvalue distribution of $H_{W}$ in the dynamical fermion simulation with two flavors of the overlap fermion. Our algorithm of the Hybrid Monte Carl update follows that of [8]. Namely, we adopt the Zolotarev rational approximation [14] with degree $N_{p}=20$ to approximate the sign function. The low-lying eigenmodes of $H_{W}$ is calculated using the implicitly restarted Lanczos method. When the lowest eigenmode changes its sign, the value of the pseudofermion action changes discontinuously. Then the reflection or refraction occurs depending on the energy of the momentum of the mode parpendicular to the surface corresponding to that eigenmode. The occurrence of the reflection and refraction is monitored during the HMC update.

Numerical simulation is performed on $4^{4}$ lattices, with overlap fermions at $m=0.2$ and $s=0.6$. Configurations are generated with $\delta t=0.02$ and an unit length of trajectory. The values of $\beta$ and $1 / \varepsilon$ are listed in Table 1. Since the lattice spacing is unknown at this stage and the lattice volume is so small, we cannot draw any definite conclusion, but we would report the status of our study.

The average number of occurrence of reflection is listed in Table 1, and the refraction is used to trace the change of topological charge, as shown below. If the topology conserving action properly works, appearance of the near-zero mode should be suppressed, and hence the reflection/refraction should also be suppressed. Our result does not show clear tendency supporting this expectation. 

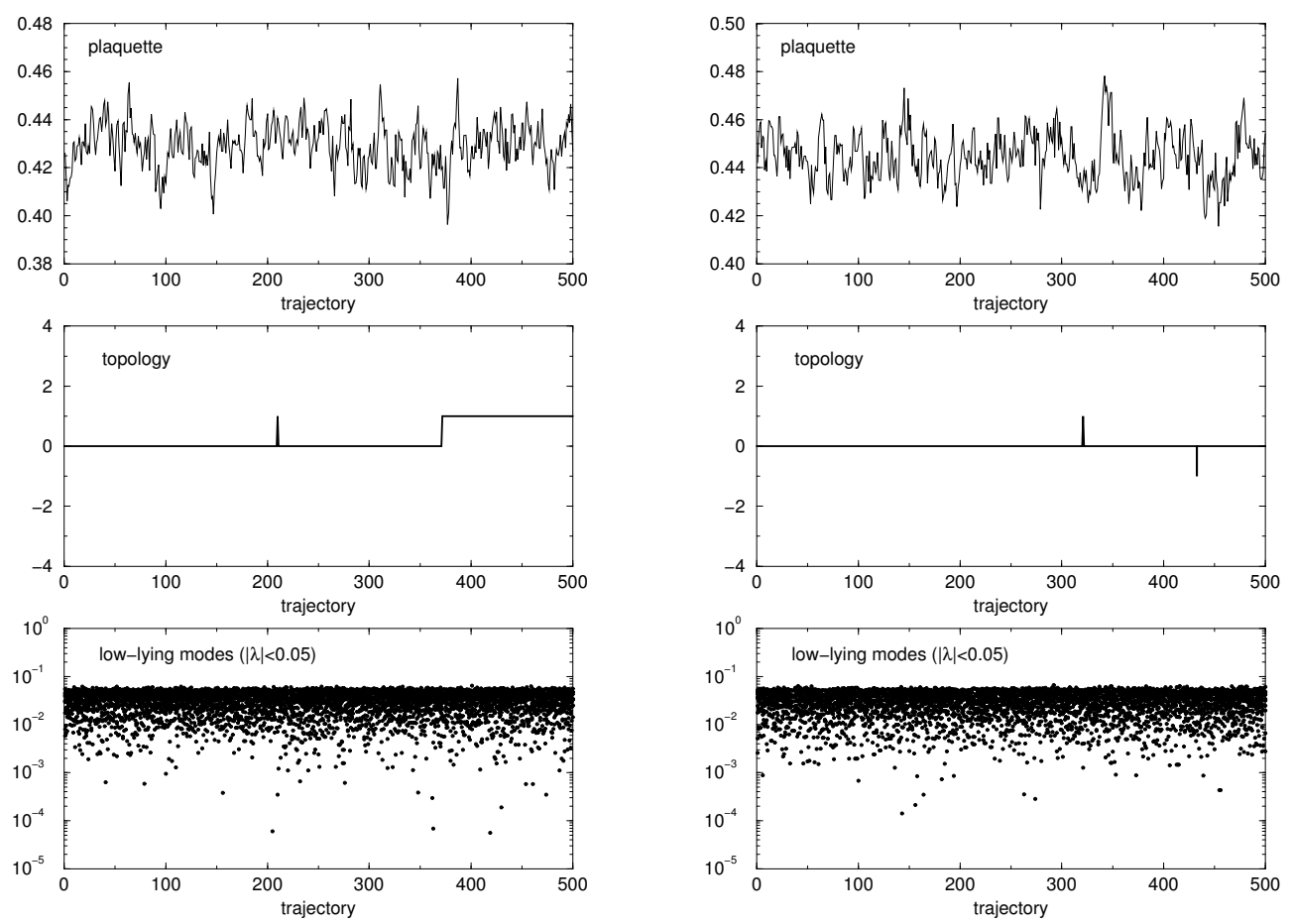

Figure 3: History of plaquette (top), topological charge (middle), and the low-lying eigenvalues (bottom). Results for the plaquette gauge action $(1 / \varepsilon=0)$ at $\beta=5.40$ (left panel) and $\beta=5.50$ (right).

The simulation time history of the plaquette, the topological charge, and the low-lying eigenvalues of $H_{W}$ is displayed in Figures 3 and $\emptyset$ for $1 / \varepsilon=0$ and 1 , respectively. The topological charge is observed by monitoring zero-crossing of the lowest eigenvalue of $H_{W}$. No clear difference is found for the topology change between the plaquette and the topology conserving gauge actions. For the distribution of the low-lying eigenmodes, the topology conserving gauge action exhibit smaller density than the plaquette action.

To draw definite conclusions, we of course need to compare these actions on fixed lattice spacings on larger lattices. The present runs are probably done on rather coarse lattices. Whether the topology conserving action is practically feasible or not will be examined in more detail.

This work is partly supported by the Large Scale Simulation Program No. 136 (FY2005) of High Energy Accelerator Research Organization (KEK). H.M. is supported by Grant-in-Aid of the Ministry of Education (No. 16740156).

\section{References}

[1] H. Neuberger, Exactly massless quarks on the lattice, Phys. Lett. B 417 (1998) 141, [hep-lat/9707022].

[2] P. Hernandez, K. Jansen and M. Luscher, Locality properties of Neuberger's lattice Dirac operator, Nucl. Phys. B552 (1999) 363, [hep-lat/9808010].

[3] R. G. Edwards, U. M. Heller and R. Narayanan, Spectral flow, chiral condensate and topology in lattice QCD, Nucl. Phys. B535 (1998) 403, [hep-lat/9802016].

[4] M. Golterman and Y. Shamir, Localization in lattice QCD, Phys. Rev. D 68 (2003) 074501, [hep-lat/0306002]. 

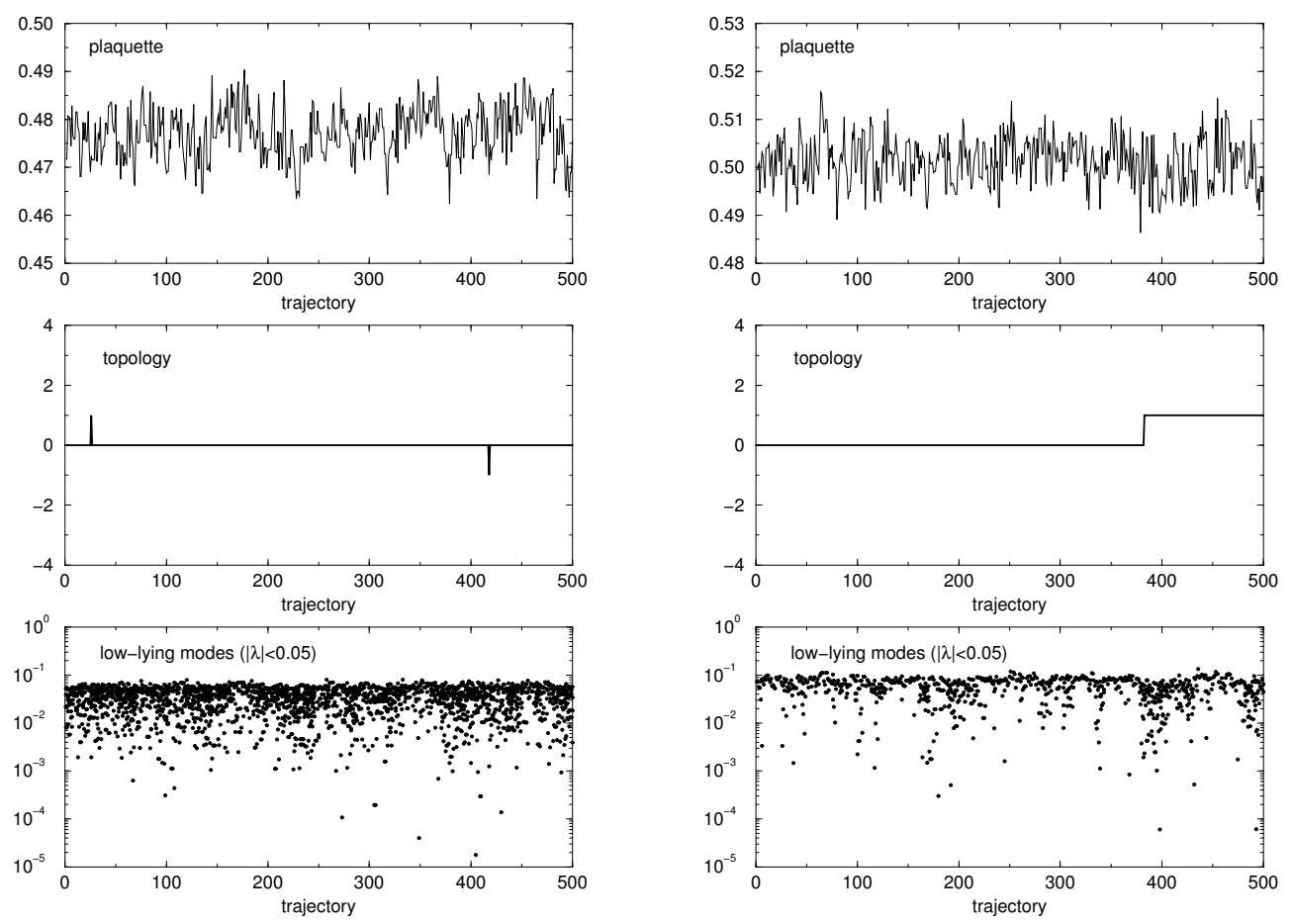

Figure 4: Same as Figure 3 , but for $1 / \varepsilon=1: \beta=0.70$ (left panel) and $\beta=0.80$ (right).

[5] M. Golterman, Y. Shamir and B. Svetitsky, Mobility edge in lattice QCD, Phys. Rev. D 71 (2005) 071502, [hep-lat/0407021].

[6] M. Golterman, Y. Shamir and B. Svetitsky, Localization properties of lattice fermions with plaquette and improved gauge actions, Phys. Rev. D 72 (2005) 034501, [hep-lat/0503037].

[7] M. Luscher, Abelian chiral gauge theories on the lattice with exact gauge invariance, Nucl. Phys. B549 (1999) 295, [hep-lat/9811032].

[8] Z. Fodor, S. D. Katz and K. K. Szabo, Dynamical overlap fermions, results with hybrid Monte-Carlo algorithm, JHEP 0408 (2004) 003, [hep-lat/0311010].

[9] H. Neuberger, Bounds on the Wilson Dirac operator, Phys. Rev. D 61 (2000) 085015, [hep-lat/9911004].

[10] H. Fukaya and T. Onogi, Lattice study of the massive Schwinger model with Theta term under Luescher's 'admissibility' condition, Phys. Rev. D 68 (2003) 074503, [hep-lat/0305004].

[11] S. Shcheredin, W. Bietenholz, K. Jansen, K. I. Nagai, S. Necco and L. Scorzato, Testing a topology conserving gauge action in QCD, hep-lat/0409073.

[12] W. Bietenholz, K. Jansen, K. I. Nagai, S. Necco, L. Scorzato and S. Shcheredin [XLF Collaboration], Lattice gauge actions for fixed topology, AIP Conf. Proc. 756 (2005) 248, [hep-lat/0412017].

[13] H. Fukaya, T. Onogi, S. Hashimoto, T. Hirohashi, and K. Ogawa, Parameter dependence of the topology change and the scaling properties of the topology conserving gauge action, $\operatorname{PoS}($ LAT2005)317.

[14] J. van den Eshof, A. Frommer, T. Lippert, K. Schilling and H. A. van der Vorst, Numerical methods for the QCD overlap operator. I: Sign-function and error bounds, Comput. Phys. Commun. 146 (2002) 203, [hep-lat/0202025]. 